\title{
Finite Element Method for Stochastic Extended KdV Equations
}

\author{
Anna Karczewska ${ }^{1 *}$, Maciej Szczeciński ${ }^{1}$, Piotr Rozmej $^{2^{* *}}$, Bartosz Boguniewicz ${ }^{2}$ \\ ${ }^{1}$ Faculty of Mathematics, Computer Science and Econometrics \\ University of Zielona Góra, Szafrana 4a, 65-246 Zielona Góra, Poland \\ *E-mail: a.karczewska@wmie.uz.zgora.pl \\ ${ }^{2}$ Faculty of Physics and Astronomy, Institute of Physics \\ University of Zielona Góra, Szafrana 4a, 65-246 Zielona Góra, Poland \\ **E-mail: p.rozmej@if.uz.zgora.pl
}

Received: 12 September 2015; revised: 26 January 2016; accepted: 29 January 2016; published online: 16 March 2016

\begin{abstract}
The finite element method is applied to obtain numerical solutions to the recently derived nonlinear equation for shallow water wave problem for several cases of bottom shapes. Results for time evolution of KdV solitons and cnoidal waves under stochastic forces are presented. Though small effects originating from second order dynamics may be obscured by stochastic forces, the main waves, both cnoidal and solitary ones, remain very robust against any distortions.
\end{abstract}

Key words: shallow water wave problem, nonlinear equations, second order $\mathrm{KdV}$ equations, stochastic forces

\section{INTRODUCTION}

The Korteveg-de Vries (KdV) equation appears as a model for the propagation of weakly nonlinear dispersive waves in several fields like gravity driven waves on a surface of an incompressible irrotational inviscid fluid [7-10, 15], ion acoustic waves in plasma [10], impulse propagation in electric circuits [15] and so on.

In hydrodynamical context the KdV equation is obtained as first order approximation of Euler equations with appropriate boundary conditions for the surface and the flat bottom. Small parameters, like $\alpha=a / H$, ratio of the wave amplitude $a$ to the fluid depth $H$ and $\beta=(H / L)^{2}$, where $L$ is a mean wavelength, are introduced. In the derivation of $\mathrm{KdV}$ the velocity potential of the fluid is expanded as a series with respect to the vertical coordinate and then only terms up to first order in small parameters are retained in all equations describing the system. Despite of its simplicity, KdV equation appeared to be a good approximation for many phenomena, see e.g. $[7,8,10,15]$ and focused enormous attention of physicists, mathematicians and engineers.
In order to extend possible applications of this kind of theories to the cases when parameters $\alpha, \beta$ are not very small the second order approximation of velocity potential was considered and subsequently second order $\mathrm{KdV}$ type equation (sometimes called extended $\mathrm{KdV}$ ) was derived, see, e.g. [3, 9]. This equation, written in scaled coordinates and fixed reference frame has the following form $(\eta(x, t)$ is the surface wave function)

$$
\begin{aligned}
\eta_{t} & +\eta_{x}+\alpha \frac{3}{2} \eta \eta_{x}+\beta \frac{1}{6} \eta_{3 x}-\frac{3}{8} \alpha^{2} \eta^{2} \eta_{x} \\
& +\alpha \beta\left(\frac{23}{24} \eta_{x} \eta_{2 x}+\frac{5}{12} \eta \eta_{3 x}\right)+\frac{19}{360} \beta^{2} \eta_{5 x}=0
\end{aligned}
$$

Here and in the following the indexes denote partial derivatives, that is $\eta_{x} \equiv \frac{\partial \eta}{\partial x}, u_{t} \equiv \frac{\partial \eta}{\partial t}$ and so on.

There were also attempts to extend the KdV theory to cases when the bottom of the fluid container is not flat. This subject is extremely important to understand the behaviour of waves coming to shallower regions and such phenomenon as tsunami creation. However, until last year there were no studies in this direction which could directly incorporate terms 
from the bottom function into the wave equation. In $[1,2]$, some of us derived second order $\mathrm{KdV}$ type equation containing terms directly related to the bottom function $h(x)$

$$
\begin{aligned}
\eta_{t} & +\eta_{x}+\alpha \frac{3}{2} \eta \eta_{x}+\beta \frac{1}{6} \eta_{3 x} \\
& -\frac{3}{8} \alpha^{2} \eta^{2} \eta_{x}+\alpha \beta\left(\frac{23}{24} \eta_{x} \eta_{2 x}+\frac{5}{12} \eta \eta_{3 x}\right)+\frac{19}{360} \beta^{2} \eta_{5 x} \\
& +\beta \delta\left(-\frac{1}{2 \beta}(h \eta)_{x}+\frac{1}{4}\left(h_{2 x} \eta\right)_{x}-\frac{1}{4}\left(h \eta_{2 x}\right)_{x}\right)=0
\end{aligned}
$$

where $\delta=a_{h} / H$ is another small parameter, $a_{h}$ stands for the amplitude of the bottom variations and $H$ is the undisturbed (mean) depth of the fluid. It is assumed that $h(x)$ is continuous, at least three times differentiable and $\lim _{x \rightarrow \pm \infty} h(x)=0$. For details of derivation of that equation, see $[1,2]$. Note that when the bottom is flat, $\delta=0$, the equation (2) reduces to (1).

The present paper aims to find numerical solutions to stochastic versions of second order KdV type equations (1) and (2). In order to do this we first try to follow the finite element method (FEM) used by Debussche and Printems in [5]. Their method was good enough for the the stochastic Korteweg-de Vries equation of the form [5, Eq. 1.2]

$$
u_{t}+u u_{x}+\epsilon u_{x x x}=\gamma \dot{\xi} .
$$

In Eq. (3) the noise term $\xi(x, t)$ is a Gaussian process with correlations

$$
\mathbb{E} \dot{\xi}(x, t) \dot{\xi}(y, s)=c(x-y) \delta(t-s)
$$

and $\gamma$ is the amplitude of the noise. Equation (3) with rhs equal zero is the Korteweg-de Vries equation written in a moving reference system with coordinates scaled in a particular way. This form was convenient for the authors in order to apply the finite element method (FEM) in their numerical simulation.

In the case of periodic boundary conditions the noise term $\xi$ has to be introduced in a different way. Since the Brownian motions are nowhere differentiable we have to introduce the mathematical form of (3).

Let $\left(\Omega, \mathcal{F},\left(\mathcal{F}_{t}\right)_{t \geq 0}, \mathbb{P}\right)$ denote a stochastic basis. The Itô form of (3) can be written in the form

$$
d u+\left(u \frac{\partial u}{\partial x}+\epsilon \frac{\partial^{3} u}{\partial x^{3}}\right) d t=\gamma \Phi d W .
$$

In (5), $W$ is a Wiener process on $L^{2}(0, L)$ of the form

$$
W(t, x)=\sum_{i=0}^{\infty} \beta_{i}(t) e_{i}(x),
$$

where $\left\{e_{i}\right\}_{i \in \mathbb{N}}$ is an orthonormal basis of $L^{2}(0, L)$ and $\left\{\beta_{i}\right\}_{i \in \mathbb{N}}$ is a sequence of independent real valued Brownian motions, defined on the stochastic basis. In (5), $\Phi$ is an appropriate linear map from $L^{2}(0, L)$ to $L^{2}(0, L)$. For more details, see [5].

\section{NUMERICAL APPROACH}

Our aim was to extend the approach used in [5] in order to numerically solve second order stochastic version of the equation with an uneven bottom (2)

$$
\begin{aligned}
\eta_{t} & +\eta_{x}+\alpha \frac{3}{2} \eta \eta_{x}+\beta \frac{1}{6} \eta_{3 x} \\
& -\frac{3}{8} \alpha^{2} \eta^{2} \eta_{x}+\alpha \beta\left(\frac{23}{24} \eta_{x} \eta_{2 x}+\frac{5}{12} \eta \eta_{3 x}\right)+\frac{19}{360} \beta^{2} \eta_{5 x} \\
& +\beta \delta\left(-\frac{1}{2 \beta}(h \eta)_{x}+\frac{1}{4}\left(h_{2 x} \eta\right)_{x}-\frac{1}{4}\left(h \eta_{2 x}\right)_{x}\right)=\gamma \dot{\xi} .
\end{aligned}
$$

Note that this equation, in contrast to KdV equation, has to be solved in the fixed coordinate system because transformation to a moving frame would make the bottom function time dependent.

Setting $\delta=0$ in (7) one obtains second order stochastic $\mathrm{KdV}$ type equation (that is the equation for the flat bottom)

$$
\begin{aligned}
\eta_{t} & +\eta_{x}+\alpha \frac{3}{2} \eta \eta_{x}+\beta \frac{1}{6} \eta_{3 x}-\frac{3}{8} \alpha^{2} \eta^{2} \eta_{x} \\
& +\alpha \beta\left(\frac{23}{24} \eta_{x} \eta_{2 x}+\frac{5}{12} \eta \eta_{3 x}\right)+\frac{19}{360} \beta^{2} \eta_{5 x}=\gamma \dot{\xi}
\end{aligned}
$$

which can be solved within the same algorithm.

The details of numerical scheme for solution of equations (1), (2) were described in [6]. Therefore in this paper we only briefly summarize that description emphasizing the stochastic part. We focus on (7) because in our scheme (8) is the particular case of (7) when $\delta=0$.

We adapt Crank-Nicholson scheme for time evolution using time step $\tau$. Introducing the following variables:

$$
v=\eta_{x}, \quad w=v_{x}, \quad p=w_{x}, q=p_{x}, g=h_{x x}
$$

we can write fifth order differential equation (7) as the coupled set of first order differential equations

$$
\begin{aligned}
& \eta^{n+1}-\eta^{n}-\Phi\left(W^{n+1}-W^{n}\right) \\
&+\tau \frac{\partial}{\partial x}\left[\eta^{n+\frac{1}{2}}+\frac{3 \alpha}{4}\left(\eta^{n+\frac{1}{2}}\right)^{2}+\frac{\beta}{6} w^{n+\frac{1}{2}}\right. \\
&-\frac{1}{8} \alpha^{2}\left(\eta^{n+\frac{1}{2}}\right)^{3}+\alpha \beta\left(\frac{13}{48}\left(v^{n+\frac{1}{2}}\right)^{2}\right. \\
&\left.+\frac{5}{12}\left(\eta^{n+\frac{1}{2}} w^{n+\frac{1}{2}}\right)\right)+\frac{19}{360} \beta^{2}\left(q^{n+\frac{1}{2}}\right) \\
& \frac{1}{4} \beta \delta\left(-\frac{2}{\beta}\left(h^{n+\frac{1}{2}} \eta^{n+\frac{1}{2}}\right)\right. \\
&\left.\left.+\eta^{n+\frac{1}{2}} g^{n+\frac{1}{2}}+h^{n+\frac{1}{2}} w^{n+\frac{1}{2}}\right)\right]=0, \\
& \frac{\partial}{\partial x} \eta^{n+\frac{1}{2}}-v^{n+\frac{1}{2}}=0, \\
& \frac{\partial}{\partial x} v^{n+\frac{1}{2}}-w^{n+\frac{1}{2}}=0, \\
& \frac{\partial}{\partial x} w^{n+\frac{1}{2}}-p^{n+\frac{1}{2}}=0, \\
& \frac{\partial}{\partial x} p^{n+\frac{1}{2}}-q^{n+\frac{1}{2}}=0,
\end{aligned}
$$


where

$$
\begin{array}{rlrl}
\eta^{n+\frac{1}{2}} & =\frac{1}{2}\left(\eta^{n+1}+\eta^{n}\right), & v^{n+\frac{1}{2}}=\frac{1}{2}\left(v^{n+1}+v^{n}\right), \\
w^{n+\frac{1}{2}}=\frac{1}{2}\left(w^{n+1}+w^{n}\right), & p^{n+\frac{1}{2}}=\frac{1}{2}\left(p^{n+1}+p^{n}\right), \\
q^{n+\frac{1}{2}}=\frac{1}{2}\left(q^{n+1}+q^{n}\right), & h^{n+\frac{1}{2}}=\frac{1}{2}\left(h^{n+1}+h^{n}\right), \\
g^{n+\frac{1}{2}}=\frac{1}{2}\left(g^{n+1}+g^{n}\right) . & &
\end{array}
$$$$
\text { In (10), } \eta^{n}=\eta(x, n \tau), \eta^{n+1}=\eta(x,(n+1) \tau) \text { and so on. }
$$

\section{1. Finite element method}

Since solutions to stochastic equation are not expected to be smooth, we follow the arguments given in [5] and apply Petrov-Galerkin space discretization and the finite element method. We use a piecewise linear shape function and piecewise constant test functions. We consider wave motion on the interval $x \in[0, L]$ with periodic boundary conditions. Let $N \in \mathbb{N}$, then we use a mesh $M_{\chi}$ of points $x_{j}=j \chi$, $j=0,1, \ldots, N$, where $\chi=L / N$. Let $V_{\chi}^{1}$ be a space of a picewise linear functions $\varphi(x)$, such that $\varphi(0)=\varphi(L)$, defined as

$$
\varphi_{j}(x)=\left\{\begin{array}{lll}
\frac{1}{\chi}\left(x-x_{j-1}\right) & \text { if } & x \in\left[x_{j-1}, x_{j}\right] \\
\frac{1}{\chi}\left(x_{j+1}-x\right) & \text { if } & x \in\left[x_{j}, x_{j+1}\right] \\
0 & & \text { otherwise. }
\end{array}\right.
$$

For test functions we choose the space of piecewise constant functions $\psi(x) \in V_{\chi}^{0}$, where

$$
\psi_{j}(x)=\left\{\begin{array}{lll}
1 & \text { if } & x \in\left[x_{j}, x_{j+1}\right) \\
0 & & \text { otherwise. }
\end{array}\right.
$$

Approximate solution and its derivatives may be expanded in the basis (12)

$$
\begin{array}{ll}
\eta_{\chi}^{n}(x)=\sum_{j=1}^{N} a_{j}^{n} \varphi_{j}(x), & v_{\chi}^{n}(x)=\sum_{j=1}^{N} b_{j}^{n} \varphi_{j}(x), \\
w_{\chi}^{n}(x)=\sum_{j=1}^{N} c_{j}^{n} \varphi_{j}(x), & p_{\chi}^{n}(x)=\sum_{j=1}^{N} d_{j}^{n} \varphi_{j}(x), \\
q_{\chi}^{n}(x)=\sum_{j=1}^{N} e_{j}^{n} \varphi_{j}(x) .
\end{array}
$$

Therefore in a weak formulation we can write (10) as

$$
\begin{aligned}
& \left(\eta_{\chi}^{n+1}-\eta_{\chi}^{n}, \psi_{i}\right)-\left(\Phi\left(W_{\chi}^{n+1}-W_{\chi}^{n}\right), \psi_{i}\right) \\
& +\tau\left\{\left(\partial_{x} \eta_{\chi}^{n+\frac{1}{2}}, \psi_{i}\right)+\frac{3 \alpha}{4}\left(\partial_{x}\left(\eta_{\chi}^{n+\frac{1}{2}}\right)^{2}, \psi_{i}\right)\right. \\
& +\frac{\beta}{6}\left(\partial_{x} w_{\chi}^{n+\frac{1}{2}}, \psi_{i}\right)-\frac{1}{8} \alpha^{2}\left(\partial_{x}\left(\eta_{\chi}^{n+\frac{1}{2}}\right)^{3}, \psi_{i}\right) \\
& +\alpha \beta\left[\frac{13}{48}\left(\partial_{x}\left(v_{\chi}^{n+\frac{1}{2}}\right)^{2}, \psi_{i}\right)\right. \\
& \left.+\frac{5}{12}\left(\partial_{x}\left(\eta_{\chi}^{n+\frac{1}{2}} w_{\chi}^{n+\frac{1}{2}}\right), \psi_{i}\right)\right] \\
& +\frac{19}{360} \beta^{2}\left(\partial_{x}\left(q_{\chi}^{n+\frac{1}{2}}\right), \psi_{i}\right) \\
& +\frac{1}{4} \beta \delta\left(\partial _ { x } \left[-\frac{2}{\beta}\left(h^{n+\frac{1}{2}} \eta_{\chi}^{n+\frac{1}{2}}\right)\right.\right. \\
& \left.\left.\left.+\eta_{\chi}^{n+\frac{1}{2}} g^{n+\frac{1}{2}}+h^{n+\frac{1}{2}} w_{\chi}^{n+\frac{1}{2}}\right], \psi_{i}\right)\right\}=0, \\
& \left(\partial_{x} \eta_{\chi}^{n+\frac{1}{2}}, \psi_{i}\right)-\left(v_{\chi}^{n+\frac{1}{2}}, \psi_{i}\right)=0, \\
& \left(\partial_{x} v_{\chi}^{n+\frac{1}{2}}, \psi_{i}\right)-\left(w_{\chi}^{n+\frac{1}{2}}, \psi_{i}\right)=0 \text {, } \\
& \left(\partial_{x} w_{\chi}^{n+\frac{1}{2}}, \psi_{i}\right)-\left(p_{\chi}^{n+\frac{1}{2}}, \psi_{i}\right)=0, \\
& \left(\partial_{x} p_{\chi}^{n+\frac{1}{2}}, \psi_{i}\right)-\left(q_{\chi}^{n+\frac{1}{2}}, \psi_{i}\right)=0,
\end{aligned}
$$

for any $i=1, \ldots, N$, where abbreviation $\partial_{x}$ is used for $\frac{\partial}{\partial x}$. 
Here and in the following,

$$
(f, g):=\int_{0}^{L} f(x) g(x) d x
$$

denotes the scalar product of functions.

In order to obtain a noise in space, at each time step $n$ and each node $j$ a random number $\kappa_{j, n}^{\chi, \tau}$ is computed according to a normal law and such that it forms a sequence of independent random variables. Then we can set

$$
\begin{aligned}
\Phi\left(W_{\chi}^{n+1}-W_{\chi}^{n}\right) & =\sqrt{\tau} \sum_{j=1}^{N} \frac{1}{\left\|\phi_{j}\right\|_{L^{2}(0, L)}} \kappa_{j, n}^{\chi, \tau} \phi_{j} \\
& =\sqrt{\tau} N_{\phi} \sum_{j=1}^{N} \kappa_{j, n}^{\chi, \tau} \phi_{j},
\end{aligned}
$$

where notation $N_{\phi}=\frac{1}{\left\|\phi_{j}\right\|_{L^{2}(0, L)}}$ was introduced for abbreviation.

Insertion (14) into (15) yields a system of coupled linear equations for coefficients $a_{j}^{n}, b_{j}^{n}, c_{j}^{n}, d_{j}^{n}, e_{j}^{n}$. Solution of this system supplies an approximate solution of (1) given in the mesh points $x_{j}$.

Denote

$$
\begin{aligned}
& C_{i j}^{(1)}:=\left(\varphi_{j}, \psi_{i}\right), \\
& C_{i j}^{(2)}:=\left(\varphi_{j}^{\prime}, \psi_{i}\right), \\
& C_{i j k}^{(3)}:=\left(\varphi_{j}^{\prime} \varphi_{k}+\varphi_{j} \varphi_{k}^{\prime}, \psi_{i}\right), \\
& C_{i j k l}^{(4)}:=\left(\left[\varphi_{j}^{\prime} \varphi_{k} \varphi_{l}+\varphi_{j} \varphi_{k}^{\prime} \varphi_{l}+\varphi_{j} \varphi_{k} \varphi_{l}^{\prime}\right], \psi_{i}\right)
\end{aligned}
$$

where $\varphi_{j}^{\prime}=\frac{d \varphi}{d x}\left(x_{j}\right)$. Simple integration shows that

$$
\begin{gathered}
C_{i j}^{(1)}=\left\{\begin{aligned}
\frac{1}{2} \chi & \text { if } i=j \quad \text { or } i=j-1 \\
0 & \text { otherwise, }
\end{aligned}\right. \\
C_{i j}^{(2)}=\left\{\begin{aligned}
-1 & \text { if } i=j \\
1 & \text { if } i=j-1 \\
0 & \text { otherwise. }
\end{aligned}\right.
\end{gathered}
$$

A little more complicated calculation yields

$$
C_{i j k}^{(3)}=C_{i j}^{(2)} \delta_{j k} \quad \text { and } \quad C_{i j k l}^{(4)}=C_{i j}^{2} \delta_{j k} \delta_{k l}
$$

Properties (19) allow to reduce double and triple sums arising in nonlinear terms in (15) to single ones.

The final system of nonlinear equations for coefficients $a_{j}^{n+1}, b_{j}^{n+1}, c_{j}^{n+1}, d_{j}^{n+1}, e_{j}^{n+1}$ of expansion of the solution in the basis $\left\{\phi_{i}\right\}$ has the form (for details of derivation, see [6])

$$
\begin{aligned}
& \sum_{j=1}^{N}\left\{\left(a_{j}^{n+1}-a_{j}^{n}+\sqrt{\tau} N_{\phi} \kappa_{j, n}^{\chi, \tau}\right) C_{i j}^{(1)}\right. \\
& +\tau\left[\frac{1}{2}\left(b_{j}^{n+1}+b_{j}^{n}\right) C_{i j}^{(1)}\right. \\
& +\left(\alpha \frac{3}{16}\left(a_{j}^{n+1}+a_{j}^{n}\right)^{2}+\beta \frac{1}{12}\left(c_{j}^{n+1}+c_{j}^{n}\right)\right. \\
& -\alpha^{2} \frac{1}{64}\left(a_{j}^{n+1}+a_{j}^{n}\right)^{3}+\alpha \beta \frac{13}{192}\left(b_{j}^{n+1}+b_{j}^{n}\right)^{2} \\
& +\alpha \beta \frac{5}{96}\left(a_{j}^{n+1}+a_{j}^{n}\right)\left(c_{j}^{n+1}+c_{j}^{n}\right) \\
& \left.\left.\left.+\beta^{2} \frac{19}{720}\left(e_{j}^{n+1}+e_{j}^{n}\right)\right) C_{i j}^{(2)}\right]\right\}=0, \\
& \sum_{j=1}^{N}\left[\left(a_{j}^{n+1}+a_{j}^{n}\right) C_{i j}^{(2)}-\left(b_{j}^{n+1}+b_{j}^{n}\right) C_{i j}^{(1)}\right]=0, \\
& \sum_{j=1}^{N}\left[\left(b_{j}^{n+1}+b_{j}^{n}\right) C_{i j}^{(2)}-\left(c_{j}^{n+1}+c_{j}^{n}\right) C_{i j}^{(1)}\right]=0, \\
& \sum_{j=1}^{N}\left[\left(c_{j}^{n+1}+c_{j}^{n}\right) C_{i j}^{(2)}-\left(d_{j}^{n+1}+d_{j}^{n}\right) C_{i j}^{(1)}\right]=0, \\
& \sum_{j=1}^{N}\left[\left(b_{j}^{n+1}+b_{j}^{n}\right) C_{i j}^{(2)}-\left(e_{j}^{n+1}+e_{j}^{n}\right) C_{i j}^{(1)}\right]=0,
\end{aligned}
$$

where $i=1,2, \ldots, N$.

Define $5 N$-dimensional vector of expansion coefficients (14)

$$
X^{n}=\left(\begin{array}{c}
A^{n} \\
B^{n} \\
C^{n} \\
D^{n} \\
E^{n}
\end{array}\right),
$$

where

$$
\begin{gathered}
A^{n}=\left(\begin{array}{c}
a_{1}^{n} \\
a_{2}^{n} \\
\vdots \\
a_{N}^{n}
\end{array}\right), B^{n}=\left(\begin{array}{c}
b_{1}^{n} \\
b_{2}^{n} \\
\vdots \\
b_{N}^{n}
\end{array}\right), C^{n}=\left(\begin{array}{c}
c_{1}^{n} \\
c_{2}^{n} \\
\vdots \\
c_{N}^{n}
\end{array}\right), \\
D^{n}=\left(\begin{array}{c}
d_{1}^{n} \\
d_{2}^{n} \\
\vdots \\
d_{N}^{n}
\end{array}\right), \quad \text { and } E^{n}=\left(\begin{array}{c}
e_{1}^{n} \\
e_{2}^{n} \\
\vdots \\
e_{N}^{n}
\end{array}\right) .
\end{gathered}
$$

In (20), $A^{n+1}, B^{n+1}, C^{n+1}, D^{n+1}, E^{n+1}$ represent the unknown coefficients whereas $A^{n}, B^{n}, C^{n}, D^{n}, E^{n}$ the known ones. Note that the system (20) is a nonlinear one.

In an abbreviated form the set (20) can be written as

$$
F_{i}\left(X^{n+1}, X^{n}\right)=0, \quad i=1,2, \ldots, 5 N .
$$


Since this equation is nonlinear we can use Newton method at each time step. That is, we find $X^{n+1}$ by iterating the equation

$$
\left(X^{n+1}\right)_{m+1}=\left(X^{n+1}\right)_{m}+J^{-1}\left(X^{n+1}\right)_{m}=0,
$$

where $J^{-1}$ is the inverse of the Jacobian of the $F\left(X^{n+1}, X^{n}\right)$ (22). Choosing $\left(X^{n+1}\right)_{0}=X^{n}$ we usually obtain the approximate solution to (22), $\left(X^{n+1}\right)_{m}$ in $m=3-5$ iterations with very good precision. The Jacobian itself is a particular sparse matrix $(5 N \times 5 N)$ with the following block structure

$$
J=\left(\begin{array}{ccccc}
\left(A_{a}\right) & \left(A_{b}\right) & \left(A_{c}\right) & (0) & \left(A_{e}\right) \\
(C 2) & -(C 1) & (0) & (0) & (0) \\
(0) & (C 2) & -(C 1) & (0) & (0) \\
(0) & (0) & (C 2) & -(C 1) & (0) \\
(0) & (0) & (0) & (C 2) & -(C 1)
\end{array}\right)
$$

where each block $(\cdot)$ is a two-diagonal sparse $(N \times N)$ matrix. The matrix $A_{a}$ is given by

$$
A_{a}=\left(\begin{array}{ccccccc}
a_{1}^{1} & 0 & 0 & \cdots & 0 & a_{N-1}^{1} & a_{N}^{1} \\
a_{1}^{2} & a_{2}^{2} & 0 & \cdots & 0 & 0 & a_{N}^{2} \\
0 & a_{2}^{3} & a_{3}^{3} & 0 & \cdots & 0 & 0 \\
\vdots & \vdots & \vdots & \ddots & \vdots & \vdots & \vdots \\
0 & 0 & \cdots & a_{N-4}^{N-3} & a_{N-3}^{N-3} & 0 & 0 \\
0 & 0 & \cdots & 0 & a_{N-3}^{n-2} & a_{N-2}^{N-2} & 0 \\
a_{1}^{N} & 0 & \cdots & 0 & 0 & a_{N}^{N-1} & a_{N}^{n}
\end{array}\right) .
$$

In (25) the nonzero elements of $\left(A_{a}\right)$ are given by

$$
a_{j}^{i}=\frac{\partial F_{i}}{\partial a_{j}^{n+1}}
$$

where $F_{i}, i=1, \ldots, N$ is given by the first equation of the set (20). Elements in the upper right and lower left corners come from periodic boundary conditions. Matrices $\left(A_{b}\right),\left(A_{c}\right),\left(A_{e}\right)$ have the same structure as $\left(A_{a}\right)$, only elements $a_{j}^{i}$ have to be replaced, respectively, by

$$
b_{j}^{i}=\frac{\partial F_{i}}{\partial b_{j}^{n+1}}, \quad c_{j}^{i}=\frac{\partial F_{i}}{\partial c_{j}^{n+1}} \quad \text { and } \quad e_{j}^{i}=\frac{\partial F_{i}}{\partial e_{j}^{n+1}} .
$$

Matrix $\left(A_{d}\right)$ vanishes since there is no fourth order derivative in the extended $\mathrm{KdV}$ equation and $d^{n}$ does not appear in $F$.

Matrices $C 1$ and $C 2$ are constant. They are defined as $C k, k=1,2$

$$
C k=\left(\begin{array}{ccccc}
C_{11}^{(k)} & 0 & \cdots & C_{11}^{(k)} & C_{1 N}^{(k)} \\
C_{21}^{(k)} & C_{22}^{(k)} & \cdots & 0 & C_{2 N}^{(k)} \\
\vdots & \vdots & \ddots & \vdots & \vdots \\
0 & 0 & \cdots & C_{N-1 N-1}^{(k)} & 0 \\
C_{N 1}^{(k)} & 0 & \cdots & C_{N-1 N}^{(k)} & C_{N N}^{(k)}
\end{array}\right)
$$

where $C_{i j}^{(k)}$ are defined in (17) and (18).

\section{RESULTS OF SIMULATIONS}

In this section some results of numerical calculations of soliton waves with stochastic forces are presented. Simulations were performed by solving the set of equations (20) step by step. The main aim was to compare time evolution of waves described by second order $\mathrm{KdV}$-type equation with bottom dependent term with stochastic forces to those obtained without these forces (obtained in previous paper [6]). In order to do this several cases of time evolution are presented in the following convention. For each particular case of the bottom function $h(x)$ a sequence of three figures is presented in which the amplitude of the stochastic force is $0,0.001$ and 0.002 , respectively. In this way the influence of an increasing stochastic term on the wave evolution is exhibited. In all presented cases $\delta=0.2$, that is, the amplitude of the bottom variation is $20 \%$ of the average water depth. In order to avoid overlaps of the wave profiles at different time instants, the consecutive profiles are shifted vertically by 0.15 .

\section{1. Soliton waves}

In figures 1-3 we compare time evolution of the wave (initialy a KdV soliton) when the bottom function represents a wide Gaussian hump,

$$
h_{1}(x)=\delta \exp \left(-\frac{(x-40)^{2}}{72}\right) .
$$

In figures 4-6 we compare time evolution of the wave (initialy a KdV soliton) when the bottom function represents a double Gaussian hump,

$$
h_{2}(x)=\delta\left[\exp \left(-\frac{(x-30)^{2}}{4}\right)+\exp \left(-\frac{(x-48)^{2}}{4}\right)\right] .
$$

In figures 7-9 we compare time evolution of the initial $\mathrm{KdV}$ soliton when the bottom function represents an extended hump,

$$
h_{3}(x)=\delta\left(\frac{\tanh (x-27)-\tanh (x-45)}{2}\right) .
$$

In figures 10-12 time evolution of the initial KdV soliton is compared for different amplitude of the stochastic term when the bottom function represents a valley, $h_{4}(x)=$ $-h_{3}(x)$. 


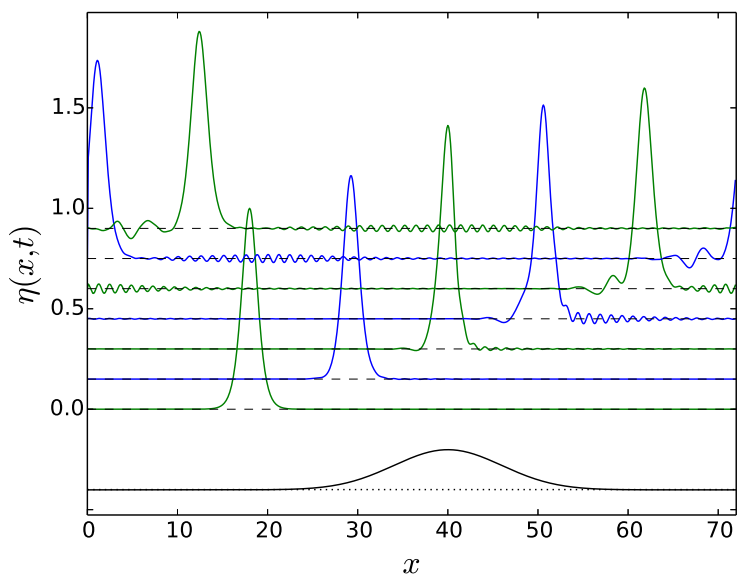

Fig. 1. Time evolution of the initial KdV soliton governed by the extended KdV equation (2) obtained with FEM method, by numerical solution of the set of equations (20) with $\gamma=0$. Dashed lines represent the undisturbed fluid surface

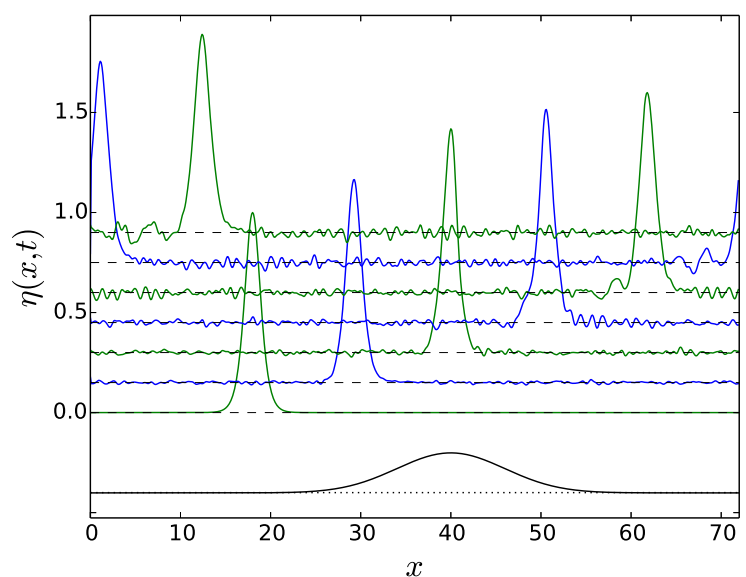

Fig. 2. The same as in Fig. 1 but with a moderate amplitude of stochastic force, $\gamma=0.001$

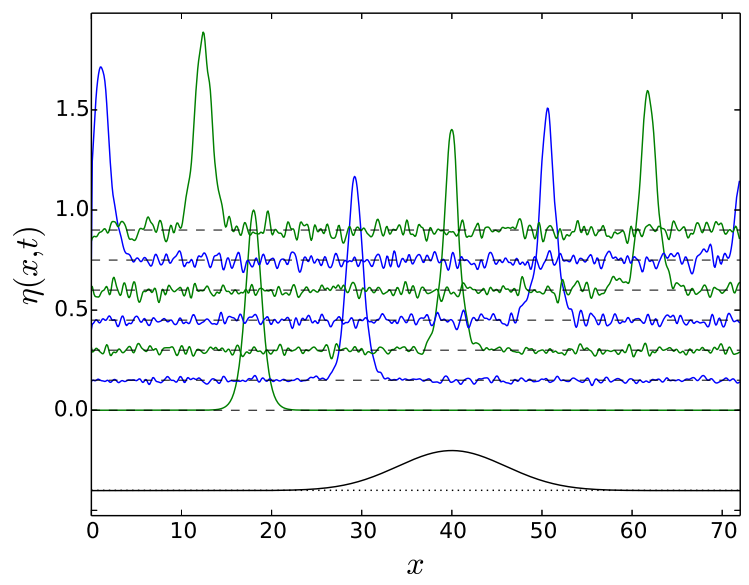

Fig. 3. The same as in Fig. 1 but with a larger amplitude of stochastic force, $\gamma=0.002$

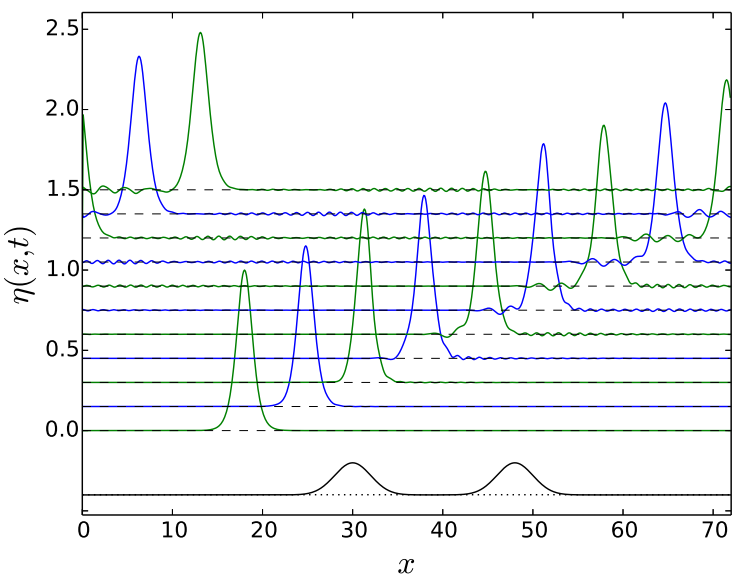

Fig. 4. The same as in Fig. 1 but for a double Gaussian hump bottom function

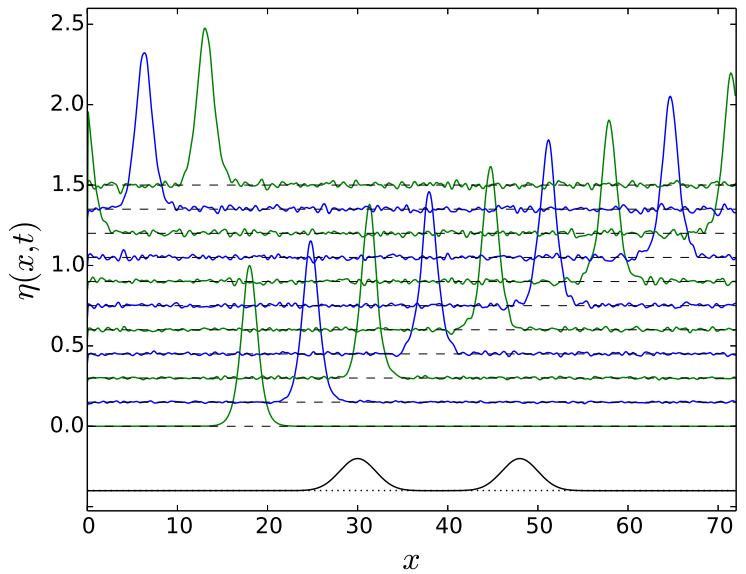

Fig. 5. The same as in Fig. 4 but with a moderate amplitude of stochastic force, $\gamma=0.001$

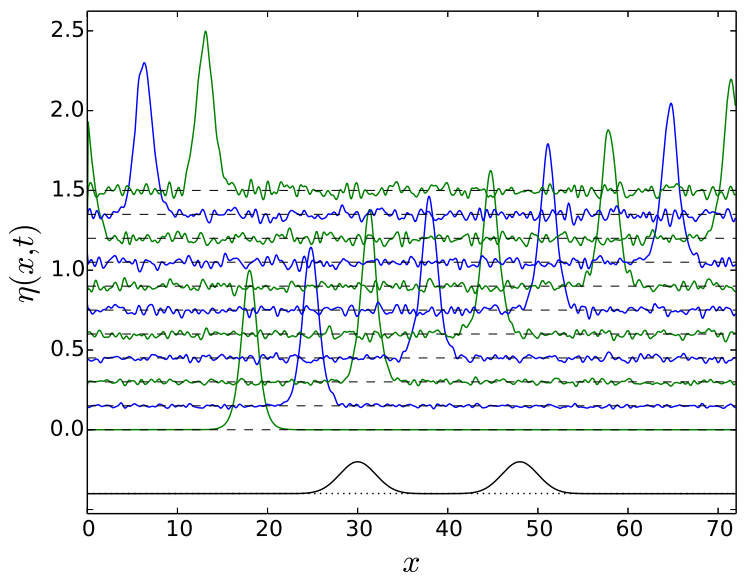

Fig. 6. The same as in Fig. 5 but with a larger amplitude of stochastic force, $\gamma=0.002$ 


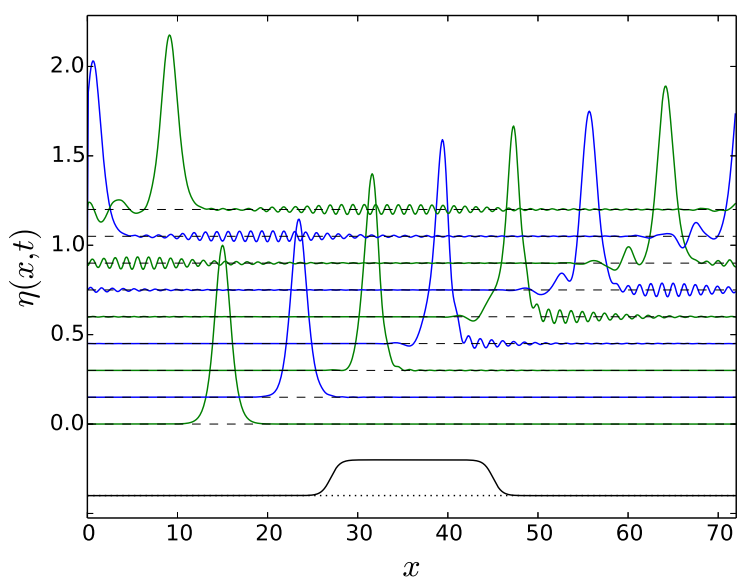

Fig. 7. The same as in Fig. 1 but for the bottom function in the form of an extended hump

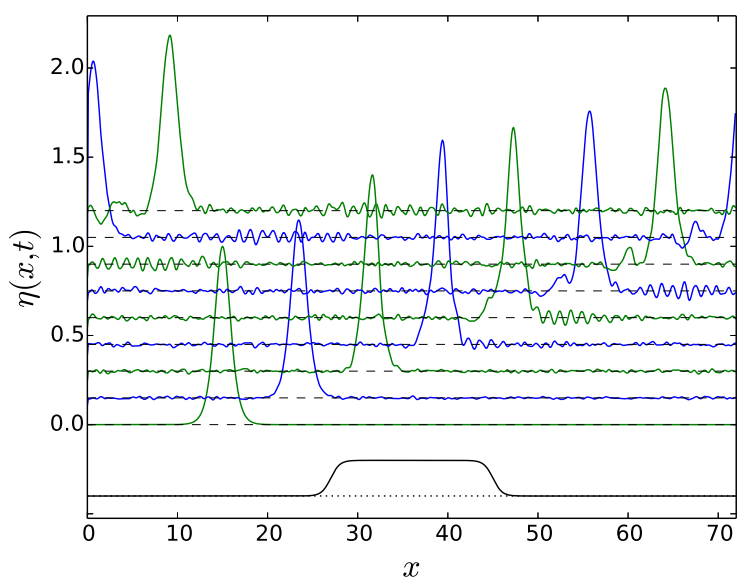

Fig. 8. The same as in Fig. 7 but with a moderate amplitude of stochastic force, $\gamma=0.001$

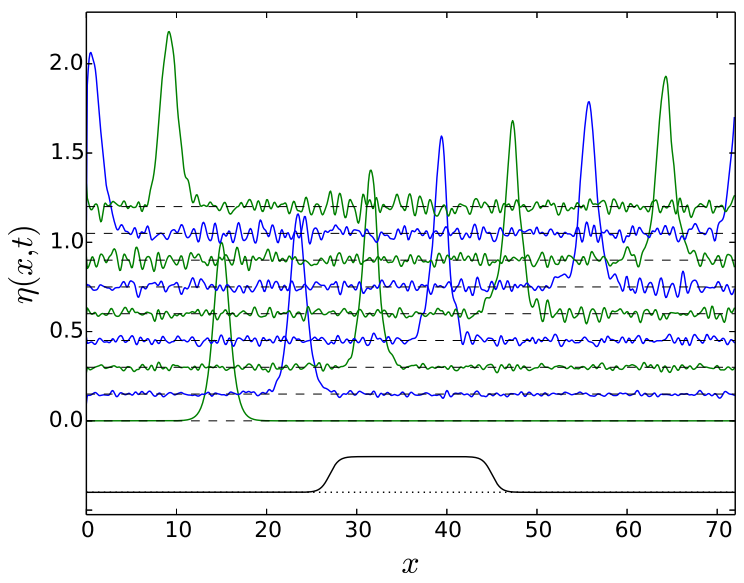

Fig. 9. The same as in Fig. 8 but with a larger amplitude of stochastic force, $\gamma=0.002$

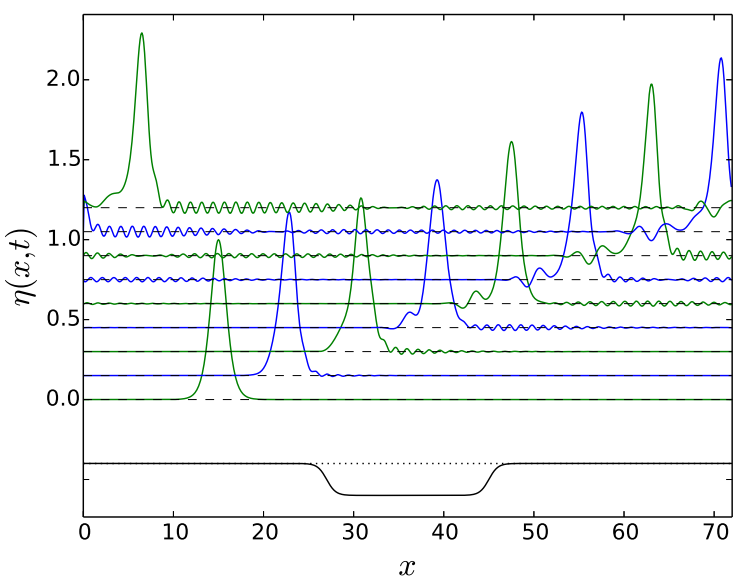

Fig. 10. The same as in Fig. 1 but for the bottom function in the form of an extended valley

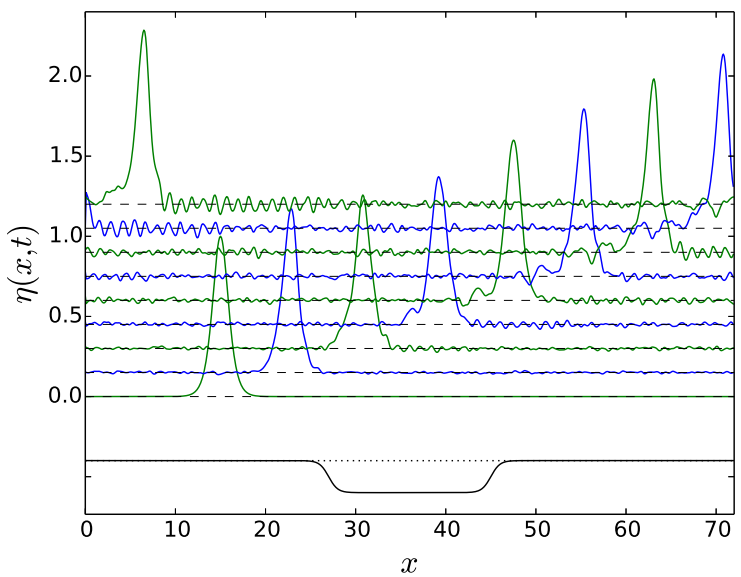

Fig. 11. The same as in Fig. 10 but with a moderate amplitude of stochastic force, $\gamma=0.001$

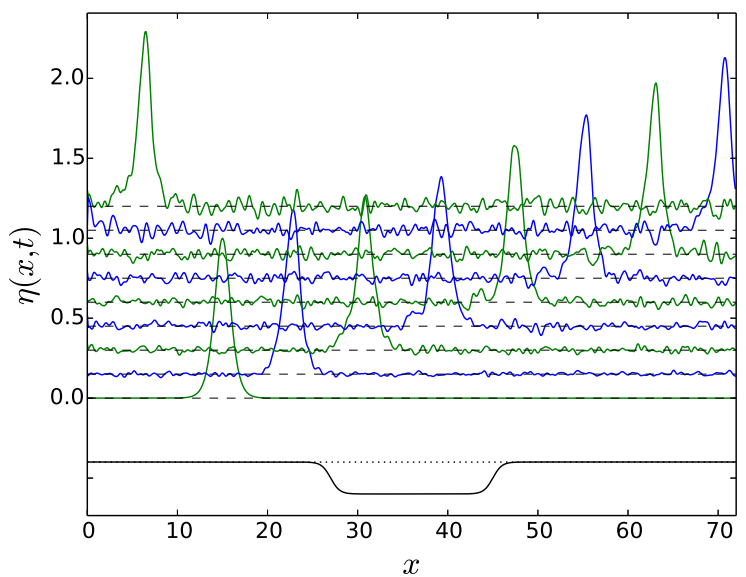

Fig. 12. The same as in Fig. 8 but with a larger amplitude of stochastic force, $\gamma=0.002$ 
In all the examples presented, with different shapes of bottom functions, one observes the same general trend. When the amplitude of the stochastic force is relatively small $(\gamma=0.001)$, some small structures originated from second order terms in the evolution equation (2) can be still visible. Simultaneously, the main soliton wave is disturbed only a little by the stochastic term.

When the amplitude of the stochastic force increases, through $\gamma=0.0015$ which case is not shown here, to $\gamma=0.002$, the structures from second order terms begin to be more and more obscured by the noise and are not visible at $\gamma=0.002$. The main wave, however, appears to be strongly resistant to the noise and preserves its soliton character.

This character is preserved also for times much longer than in presented examples. To see that we needed, however, to adapt our different code, based on finite difference method, to the stochastic case. That code proved to be very efficient in numerical calculations presented in $[1,2,4]$. The reasons why the finite difference method is more effective than finite element method presented here are explained in detail in the next section.

\section{2. Cnoidal waves}

The cnoidal solutions to KdV are expressed by the Jacobi elliptic $\mathrm{cn}^{2}$ function. The explicit expression for such a solution is the following, see, e.g. [23]:

$$
\eta(x, t)=\eta_{2}+H \mathrm{cn}^{2}\left(\frac{x-c t}{\Delta} \mid m\right),
$$

where

$$
\eta_{2}=\frac{H}{m}\left(1-m-\frac{E(m)}{K(m)}\right), \quad \Delta=h \sqrt{\frac{4 m h}{3 H}},
$$

and

$$
c=\sqrt{g h}\left[1+\frac{H}{m h}\left(1-\frac{m}{2}-\frac{3 E(m)}{2 K(m)}\right)\right] .
$$

Solution (28)-(30) is written in dimensional quantities, where $H$ is the wave height, $h$ is mean water depth, $g$ is gravitational acceleration and $m$ is an elliptic parameter. $K(m)$ and $E(m)$ are complete elliptic integrals of the first and the second kind, respectively. The value of $m \in[0,1]$ governs the shape of the wave. When $m \rightarrow 1$ the solution tends to a soliton wave with distance between the peaks going to infinity. When $m \rightarrow 0$ the cnoidal solution tends to usual sinusoidal wave.

For our equations (2) and (1) we have to express the formulas (28)-(30) in dimensionless variables.

In figures 13-15 we display time evolution of the wave, initialy cnoidal solution of KdV equation with $m=1-10^{-8}$ for $\gamma=0,0.001$ and 0.0015 . In this case the bottom function is $h(x)=\delta \frac{1}{2}\left[-\tanh \left(2(x-8.6)-\frac{1}{2}\right)+\tanh (2(x-66.5552)-\right.$ $\left.\left.\frac{1}{2}\right)\right]$ and the wavelength of the cnoidal wave is $d \approx 40.324$.

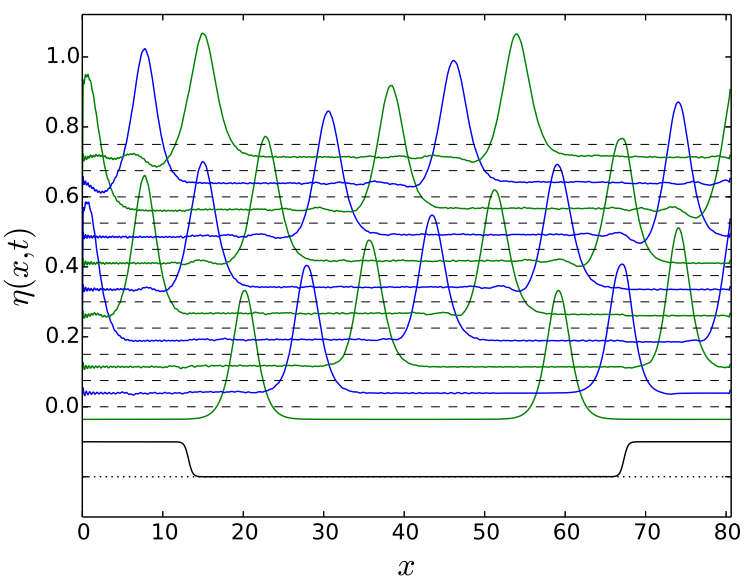

Fig. 13. Time evolution, according to eq. (2), of the cnoidal wave for the bottom function in the form of an extended valley. The $x$ interval is equal to the double wavelength of the cnoidal wave with $m=1-10^{-8}$

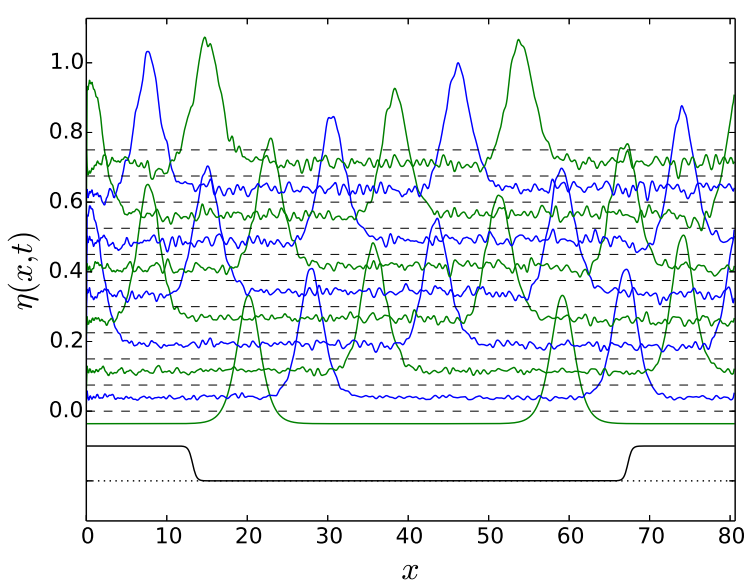

Fig. 14. The same as in Fig. 13 but with a moderate amplitude of stochastic force, $\gamma=0.001$

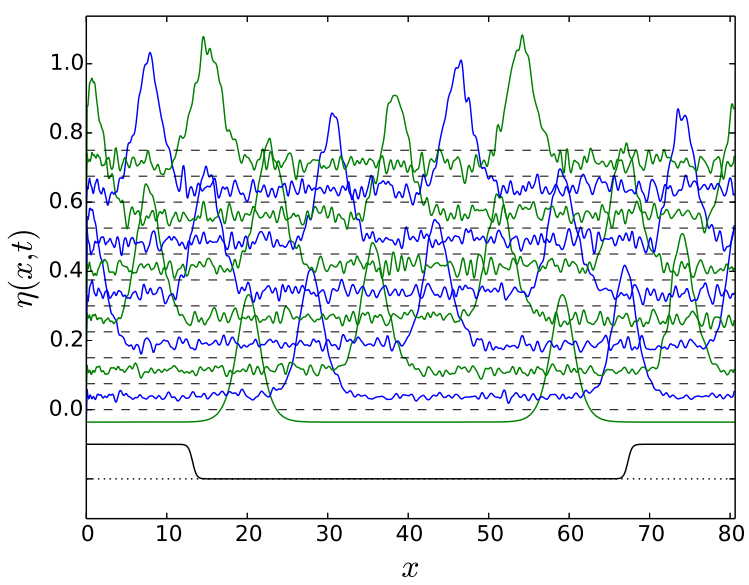

Fig. 15. The same as in Fig. 14 but with a larger amplitude of stochastic force, $\gamma=0.0015$ 


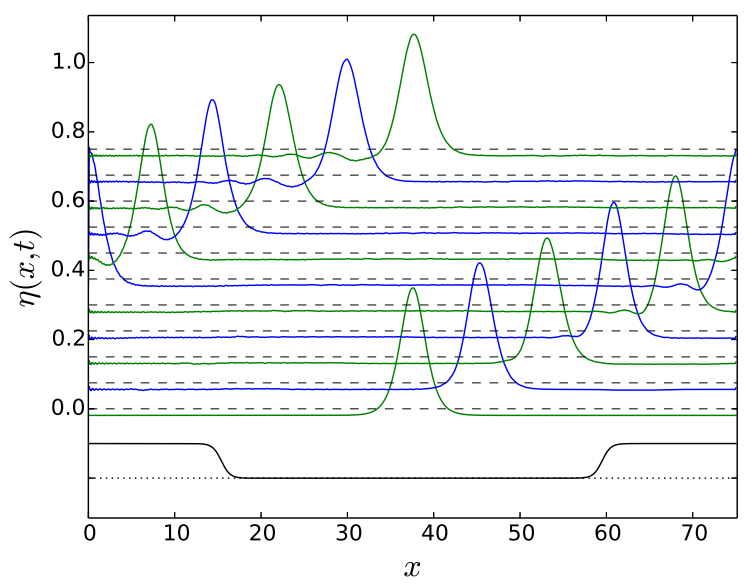

Fig. 16. Time evolution, according to eq. (2), of the cnoidal wave for the bottom function in the form of an extended valley. The $x$ interval is equal to the wavelength of the cnoidal wave with $m=1-10^{-16}$

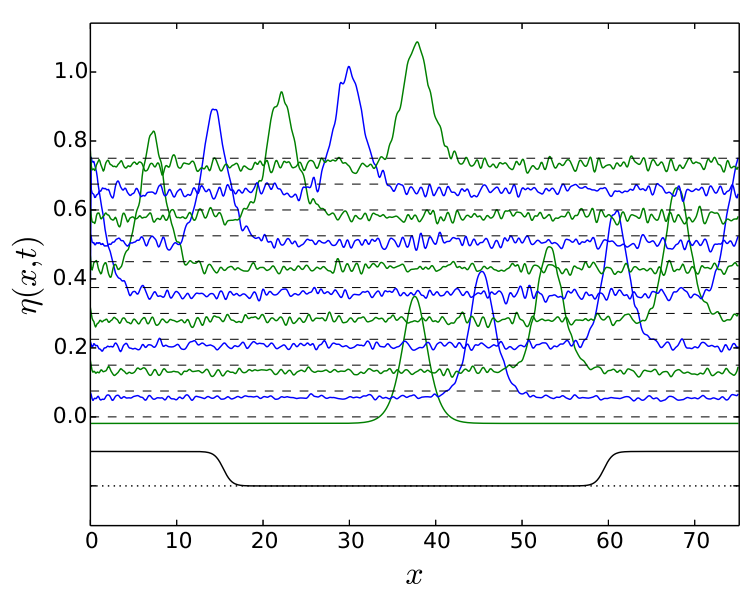

Fig. 17. The same as in Fig. 16 but with a moderate amplitude of stochastic force, $\gamma=0.001$

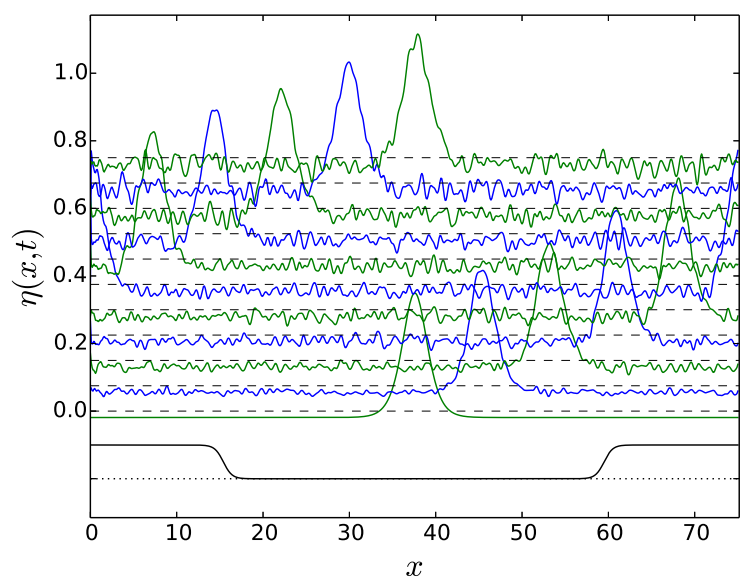

Fig. 18. The same as in Fig. 17 but with a larger amplitude of stochastic force, $\gamma=0.0015$

\section{CONCLUSIONS}

The main conclusions obtained from our numerical simulations of time evolution of $\mathrm{KdV}$-type waves with respect to second order equations with bottom terms are the following:

- Both solitary and cnoidal solutions of KdV equations are extremely robust structures for many possible distortions. In previous studies $[1,2,4]$ we showed the resistance of these waves to second order terms in extended $\mathrm{KdV}$ equation, including terms from an uneven bottom.

- In this paper we showed that an inclusion of a stochastic force into second order KdV-type equation does not disturb much the shape of the main wave even for large amplitude of the noise, although the secondary wave structures can be completely obscured by the noise. It seems, however, that the main wave is the most robust for solitary waves (which is a limitnig case of cnoidal waves when $m$ tends to 1 ). That robustness with respect to stochastic noise diminishes when parameter $m$ decreases below 1 .

- Finite element method, though sufficient for numerical study of stochastic KdV equation in [5] is not so well suited for the higher order KdV type equation, and particularly less satisfactory when the bottom is not flat. For KdV equation considered in a moving frame (3) the wave motion is slow and important time evolution can be calculated using not very long space and time intervals. This property allowed the authors of [5] to use relatively low number $N=200$ of the mesh size to obtain relevant results. Consequently, since KdV is a third order differential equation, the size of Jacobian matrix used in numerical scheme, $(3 N \times 3 N)$ is still low and allows for fast calculations. Second order ("extended") KdV equation (1), which is a differential equation of fifth order, can be studied both in a moving reference frame and in a fixed frame. In the first case the size of the Jacobian increases to $(5 N \times 5 N)$ and when $N$ is of the same order the results can still be obtained in reasonable computing time. The equation taking into account bottom variation (2), however, has to be solved in the fixed frame. Then, since waves move much faster, in order to obtain a good description of the wave evolution, substantially longer space intervals have to be used. For a resolution of fine structures of the wave relatively dense mesh has to be applied so $N$ is usually an order of magnitude larger than in the case of moving reference frame. Then computer time for inversion of the Jacobian becomes very large and detail calculations are not practical. In these cases the finite difference method used in [1,2], adapted for stochastic equation, proves to be more efficient. 


\section{References}

[1] A. Karczewska, P. Rozmej and L. Rutkowski, A new nonlinear equation in the shallow water wave problem, Physica Scripta 89, 054026 (2014).

[2] A. Karczewska, P. Rozmej and E. Infeld, Shallow-water soliton dynamics beyond the Korteweg-de Vries equation, Physical Review E 90, 012907 (2014).

[3] G.I. Burde, A. Sergyeyev, Ordering of two small parameters in the shallow water wave problem, J. Phys. A: Math. Theor. 46, 075501 (2013).

[4] A. Karczewska, P. Rozmej and E. Infeld, Energy invariant for shallow water waves and Korteweg - de Vries equation. Is energy always invariant?, arXiv:1503.09089, to be published.

[5] A. Debussche and I. Printems, Numerical simulation of the stochastic Korteweg-de Vries equation, Physica D 134, 200226 (1999).

[6] A. Karczewska, P. Rozmej, M. Szczeciński and B. Boguniewicz, Finite element method for extended $K d V$ equations, Int. J. Appl. Math. Comp. Sci. (2016), in print.

[7] P.G. Drazin and R.S. Johnson, Solitons: An Introduction, Cambridge University Press, Cambridge, 1989.

[8] G.B. Whitham, Linear and Nonlinear Waves, Wiley, New York, 1974.

[9] T.R. Marchant and N.F. Smyth, The extended Korteweg-de Vries equation and the resonant flow of a fluid over topography, J. Fluid Mech. 221, 263-288 (1990).

[10] E. Infeld and G. Rowlands, Nonlinear Waves, Solitons and Chaos, 2nd edition, Cambridge University Press, 2000.

[11] N.J. Zabusky and M.D. Kruskal, Interaction of 'Solitons' in a Collisionless Plasma and the Recurrence of Initial States, Phys. Rev. Lett. 15, 240-243 (1965).
[12] A. de Bouard, A. Debussche, On the stochastic Kortevegde Vries equation, J. Functional Analysis 154, 215-251 (1998).

[13] T. Oh, Periodic stochastic Korteweg-de Vries equation with additive space-time white noise, Anal. PDE 2, 281-304 (2009).

[14] T. Oh, Invariance of the White Noise for KdV, Commun. Math. Phys. 292, 217-236 (2009).

[15] M. Remoissenet, Waves Called Solitons: Concepts and Experiments, Springer, 1999.

[16] T.R. Marchant and N.F. Smyth, Soliton Interaction for the Korteweg-de Vries equation, IMA J. Appl. Math. 56, 157-176 (1996).

[17] T.R. Marchant, Coupled Korteweg-de Vries equations describing, to higher-order, resonant flow of a fluid over topography, Phys. Fluids 11(7), 1797-1804 (1999).

[18] Q. Zou and CH-H. Su, Overtaking collision between two solitary waves, Phys. Fluids 29, No. 7, 2113-2123 (1986).

[19] J. Villegas G., J. Castano B., J. Duarte V., E. Fierro Y., WaveletPetrov-Galerkin method for the numerical solution of the $K d V$ equation, Appl. Math. Sci. 6, 3411-3423 (2012).

[20] S.-ul-Islam, S. Haq and A. Ali, A meshfree method for the numerical solution RLW equation, J. Comp. Appl. Math. 223, 997-1012 (2009).

[21] A. Canivar, M. Sari and I. Dag, A Taylor-Galerkin finite element method for the KdV equation, Physica B 405, 3376-3383 (2010).

[22] T.R. Taha, and M.J. Ablowitz, Analytical and numerical aspects of certain nonlinear evolution equations III. Numerical, the Korteweg-de Vries equation, Journal of Computational Physics 55, 231-253 (1984).

[23] M.W. Dingemans, Water wave propagation over uneven bottoms, World Scientific, Singapore, 1997. http://repository. tudelft.nl
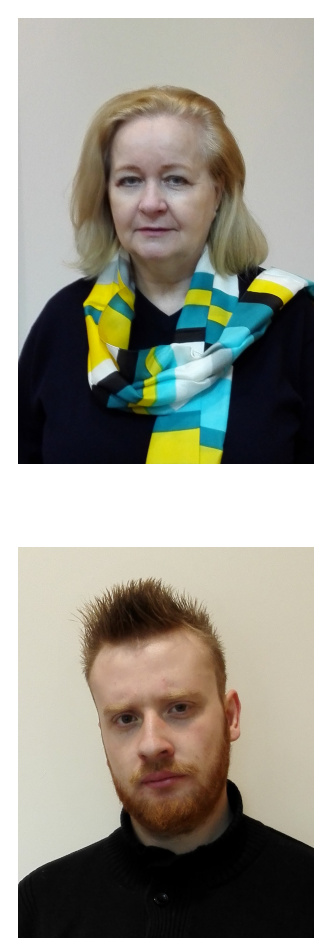

Anna Karczewska is a professor at the Faculty of Mathematics, Computer Science and Econometrics, University of Zielona Góra. She holds an MSc degree from the Warsaw Technical University, a PhD degree from Jagiellonian University, Cracow, and habilitation from the University of Zielona Góra. Her fields of interest include deterministic and stochastic integral equations, in particular Volterra equations of integral and fractional order, fluid dynamics and Korteweg-de Vries type equations.
Maciej Szczeciński holds MSc degree in economy from Wrocław University of Economics and MSc degree in mathematics obtained at Faculty of Mathematics, Informatics and Econometry, University of Zielona Góra. At present he is $\mathrm{PhD}$ student at this faculty and prepares his thesis under professor's Anna Karczewska supervision. 


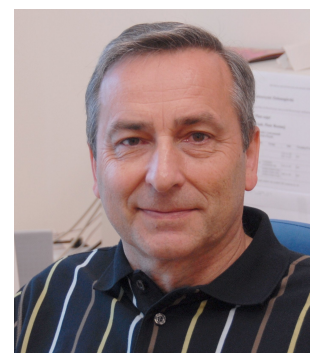

Piotr Rozmej is a full professor at the Faculty of Physics and Astronomy, University of Zielona Góra, the head of the Division of Mathematical Methods in Physics. He holds an MSc Degree from Maria Curie-Skłodowska University, Lublin, a PhD degree from Warsaw University, and habilitation from UMCS. He became a professor in 1992 and a full professor in 1997. He has spent several years working as a senior researcher at the Joint Institute for Nuclear Research (Dubna, USSR), Gesellschaft für Schwerionenforschung (Darmstadt, Germany) and Institut des Sciences Nucléaires (Grenoble, France). He worked in several fields of theoretical nuclear physics like nuclear structure, nuclear reactions, properties of heavy and superheavy nuclei. Other areas of his scientific activity include quantum optics, in particular wave packet dynamics, deterministic chaos, nonlinear dynamics, solitons, numerical modeling and simulations.

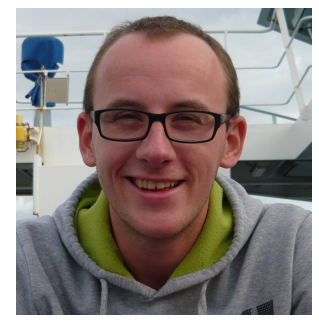

Bartosz Boguniewicz is a student at Faculty of Physics and Astronomy, University of Zielona Góra. He is finishing his master of science thesis on Korteweg-de Vries type equation under supervision of professor Piotr Rozmej. 